\title{
Polygonal Scanners: Components, Performance, and Design
}

\section{Glenn E. Stutz}

Lincoln Laser Company

Phoenix, Arizona, USA

\section{CONTENTS}

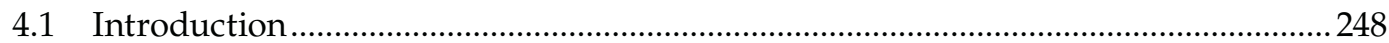

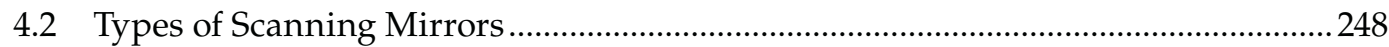

4.2.1 Prismatic Polygonal Scanning Mirrors............................................................ 249

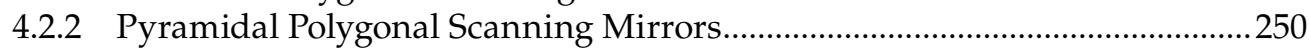

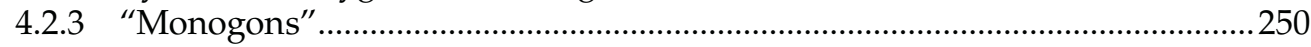

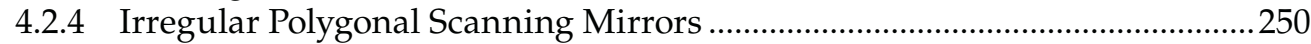

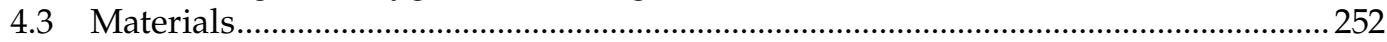

4.4 Polygonal Mirror Fabrication Techniques...............................................................253

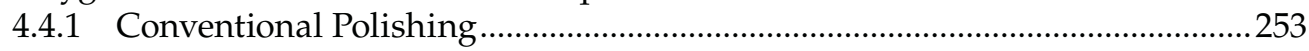

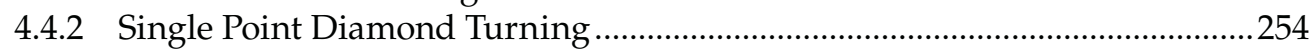

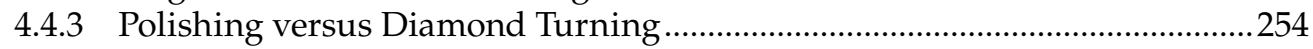

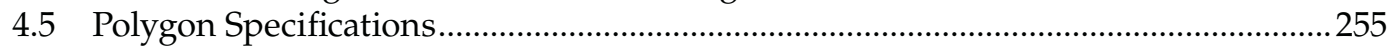

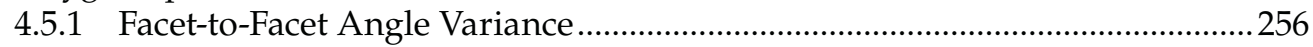

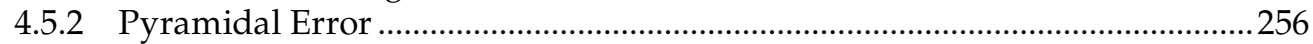

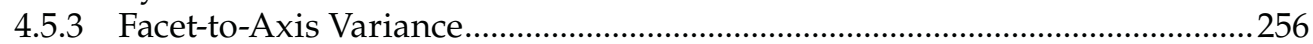

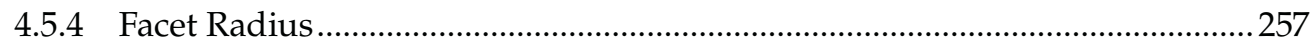

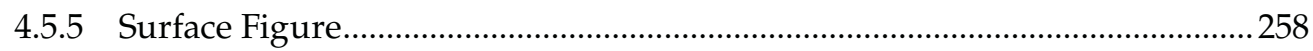

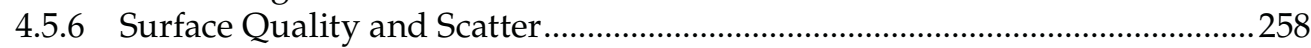

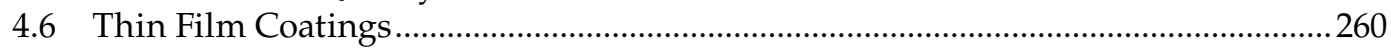

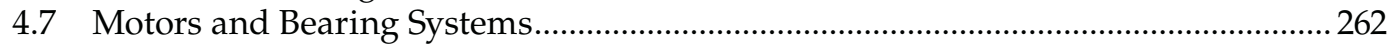

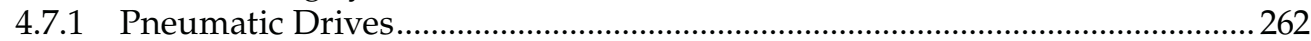

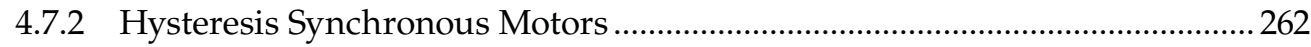

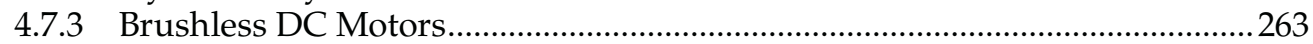

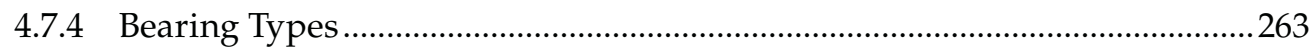

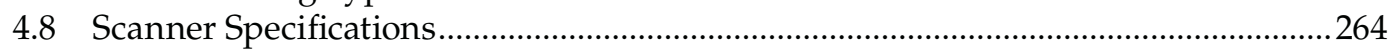

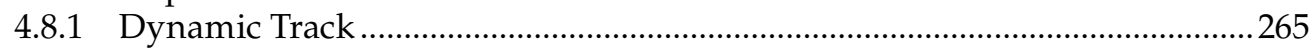

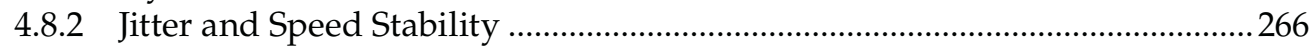

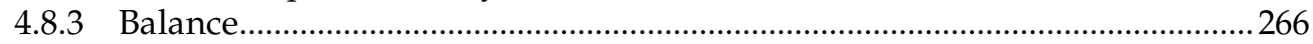

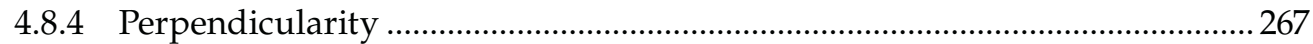

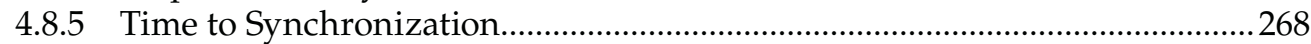

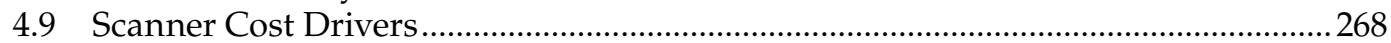

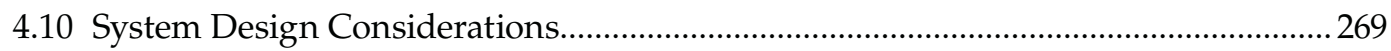

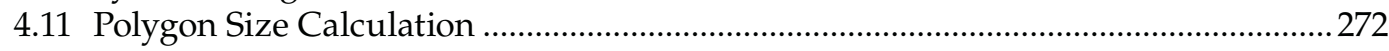


4.12 Minimizing Image Defects in Scanning Systems ................................................. 274

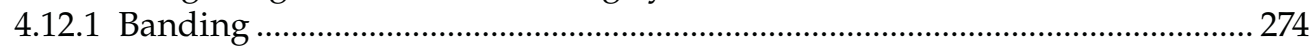

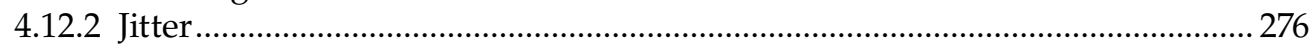

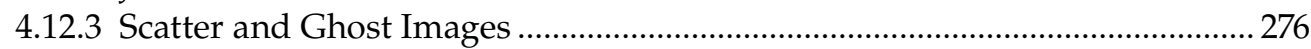

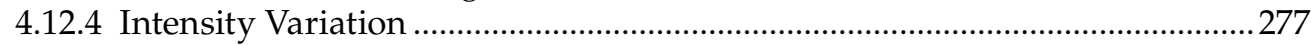

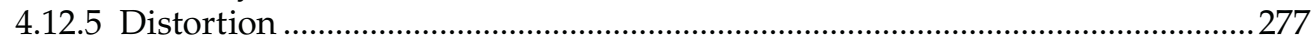

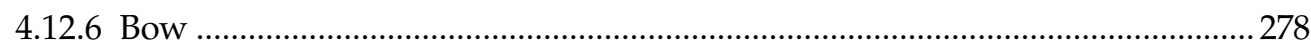

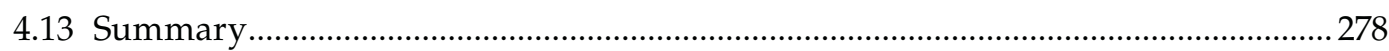

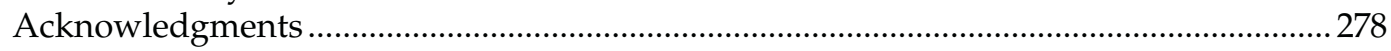

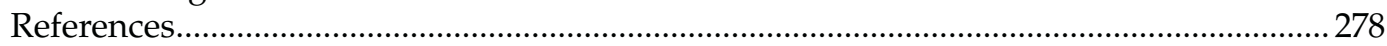

\subsection{INTRODUCTION}

Polygonal scanners have found a role in a wide range of applications including inspection, laser printing, medical imaging, laser marking, laser radar, and displays, to name a few. Ever since the laser was first discovered, engineers have needed a means to move the laser output in a repetitive format.

The term "polygonal scanner" refers to a category of scanners that incorporate a rotating optical element with three or more reflective facets. The optical element in a polygonal scanner is usually a metal mirror. In addition to the polygonal scanner other scanners can have as few as one facet such as a pentaprism, cube beam splitter, or "monogon." This section will concentrate on scanners that use a metal mirror as the optical element.

Polygonal scanners are not the only technology available to move an optical beam. These other technologies include galvanometers, micromirrors, hologons, piezo mirrors, and acousto-optic deflectors. Each technology has a niche where it excels. Polygonal scanners excel in applications requiring unidirectional scans, high scan rates, large apertures, large scan angles, or high throughputs. The polygonal scanner in most applications is paired with another means for beam steering or object motion to produce a second axis. This creates a raster image with the polygonal scanner producing the fast scan axis of motion.

This chapter will provide information on types of scan mirrors, fabrication techniques to create these mirrors, and typical specifications for these mirrors. The motor and bearing systems used with the mirror to construct a scanner are covered. A section on properly specifying a polygonal scanner as well as the cost drivers in the scanner design is included. The incorporation of the scanner into a scan system including system level specifications and design approaches is reviewed. The final section covers system image defects and methods used to compensate for these defects in a scanning system.

\subsection{TYPES OF SCANNING MIRRORS}

There are many types of scan mirrors, but most can be included in the following categories:

1. Prismatic polygonal scanning mirrors

2. Pyramidal polygonal scanning mirrors 
3. "Monogons"

4. Irregular polygonal scanning mirrors

\subsubsection{Prismatic Polygonal Scanning Mirrors}

A regular prismatic polygon is defined as one having a number of plane mirror facets that are parallel to, equidistant from, and face away from a central rotational axis (Figure 4.1). This type of scan mirror is used to produce repetitive scans over the same image plane. It is the most cost effective to manufacture and therefore finds its way into the vast majority of applications including barcode scanning and laser printing. An illustration of why the manufacturing cost can be lower than other types of scan mirrors is shown in Figure 4.2.

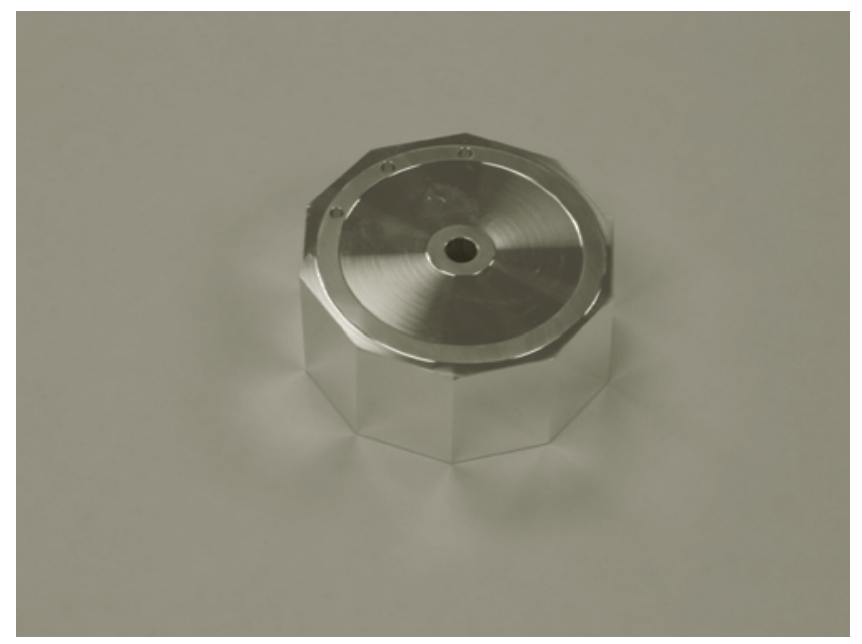

\section{FIGURE 4.1}

Regular prismatic polygonal scanning mirror.

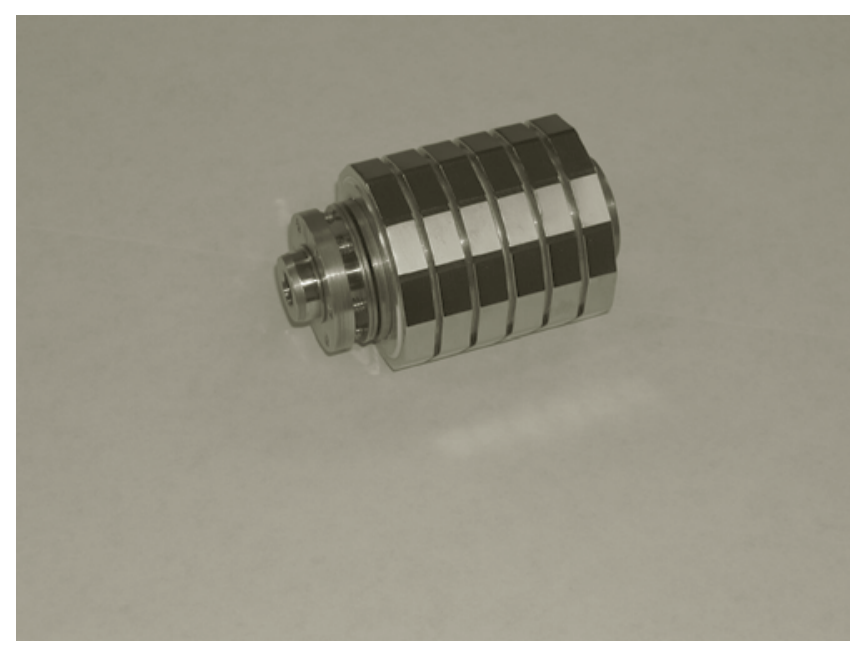

FIGURE 4.2

Mirror stack reduces fabrication costs. 
Here we see a stack of mirrors that can be moved through the manufacturing process as a single piece resulting in less handling, more consistency, and less machining time.

\subsubsection{Pyramidal Polygonal Scanning Mirrors}

A regular pyramidal polygon is defined as one having a number of facets inclined at the same angle, usually $45^{\circ}$, to the rotational axis (Figure 4.3). This type of polygon is expensive to manufacture since one cannot stack mirrors together to process at the same time as is done with regular prismatic polygons.

A significant feature of the $45^{\circ}$ pyramidal polygon is that it can produce half the output scan angle of a prismatic polygon for the same amount of shaft rotation. This feature can be used to the system designer's advantage by reducing data rates for a given polygon rotation speed. Prismatic polygons are used primarily with the input beam perpendicular to the rotation axis whereas pyramidal polygons are used primarily with the input beam parallel to the rotation axis (Figure 4.4).

\subsection{3 "Monogons"}

"Monogons" are scan mirrors where there is only one facet centered on the rotational axis. Because there is only one facet, a monogon is not a true polygon but is an important subset of the scan mirror family. Monogons are also referred to as truncated mirrors and find application in internal drum scanning. In a typical system employing a monogon, the laser is directed toward the monogon along the rotation axis and the output sweeps a circle on an internal drum as the scanner rotates. This type of scan system can produce very accurate spot placement and very high resolution and finds application in the prepress market. An example of a monogon scan mirror is shown in Figure 4.5.

\subsubsection{Irregular Polygonal Scanning Mirrors}

An irregular polygonal scanning mirror is defined as one having a number of plane facets that are at a variety of angles with respect to, and face away from, the rotational axis

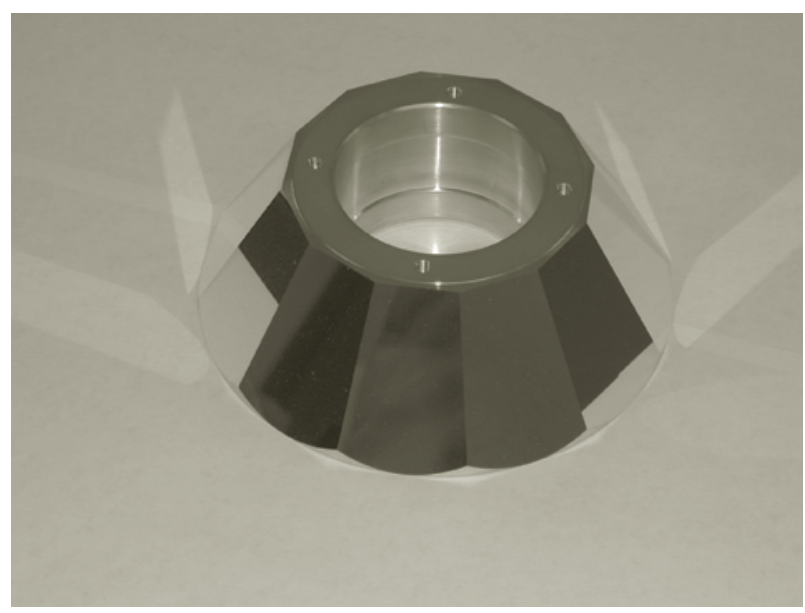

FIGURE 4.3

Regular pyramidal polygonal scanning mirror. 


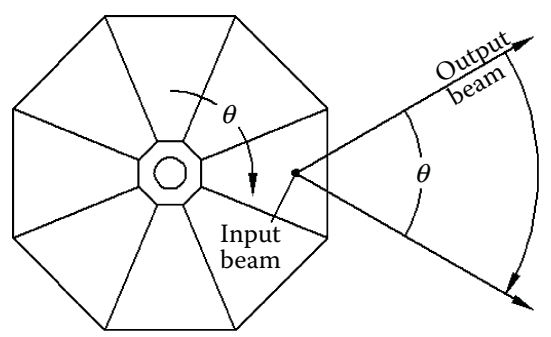

Regular $45^{\circ}$ pyramidal polygon

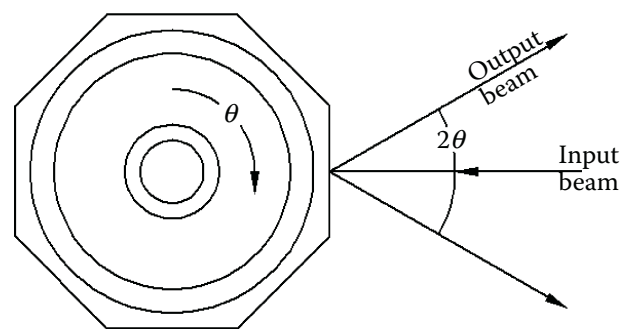

Regular prismatic polygon

FIGURE 4.4

Scan angle versus rotation angle.

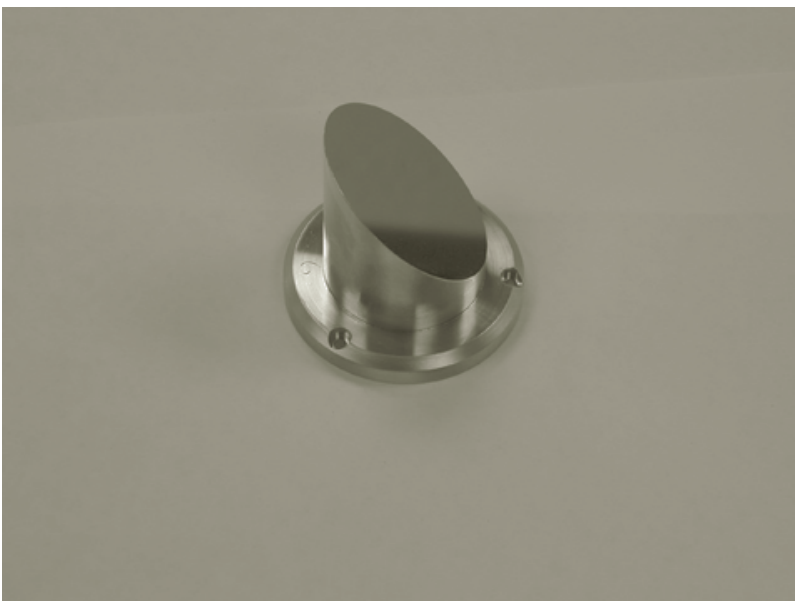

\section{FIGURE 4.5}

"Monogon."

(Figure 4.6). The unique feature of this type of scan mirror is that it can produce a raster output without a second axis of motion. The resulting output scans are nonsuperimposing if the facets are at different angles. This type of scanner finds its way into coarse scanning applications such as:

1. Point-of-sale barcode readers

2. Laser heat-treating systems

3. Low resolution writing and display systems 


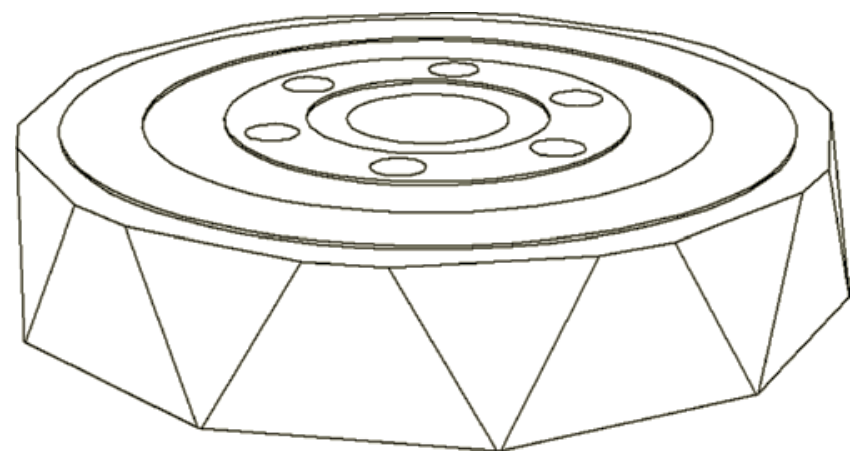

FIGURE 4.6

Irregular polygonal scanning mirror.

These polygons typically cost significantly more than regular polygons because their asymmetry prevents cost savings from stacking. Another disadvantage of these scanners is the inherent dynamic imbalance of the polygon during rotation. This limits use to low-speed applications. A special case where equal and opposing facets are used on each side of the polygon helps with the balance problem. The result is the scan pattern is generated twice each revolution.

Now that the types of scan mirrors have been covered, a logical next step is to consider the materials used to fabricate the mirrors. The following section addresses the most common materials in use today.

\subsection{MATERIALS}

Material selection for polygonal mirrors is driven by considerations of performance and cost. The most common materials for polygonal mirrors are aluminum, plastic, and beryllium. Facet distortion/flatness is a key performance consideration when choosing a material.

Aluminum represents a good trade-off between cost and performance. This material has good stiffness, is relatively light, and lends itself to low-cost fabrication methods. The upper limit for the use of aluminum mirrors without the risk of facet distortion beyond $\lambda / 10$ is on the order of a tip velocity of $76 \mathrm{~m} / \mathrm{s}$. Above this speed the size of the facet, the disc shape, and the mounting method all play a role in the distortion of the facet. It is recommended that a finite element analysis be performed if you intend to operate above this level. An example of the shape change due to high-speed rotation, for a six-faceted polygon, is shown in Figure 4.7.

Plastic is used in applications where cost is the primary concern and performance is good enough for the application. An example is in the hand-held barcode market and other short-range, low-resolution scanning applications. Injection molding techniques have come far in the past few years but it is still difficult to reliably produce plastic mirrors larger than 2-mm diameter with facets flat to better than 1 wave.

Beryllium has been used successfully in applications where high speed and low distortion are required. It is a very expensive substrate and produces toxic dust when machined, requiring specialized extraction and filtration equipment. Therefore it does not find wide usage and is a very expensive solution. Beryllium is typically nickel plated prior to polishing. The nickel plating seals in the beryllium and removes the risk of toxic dust. 


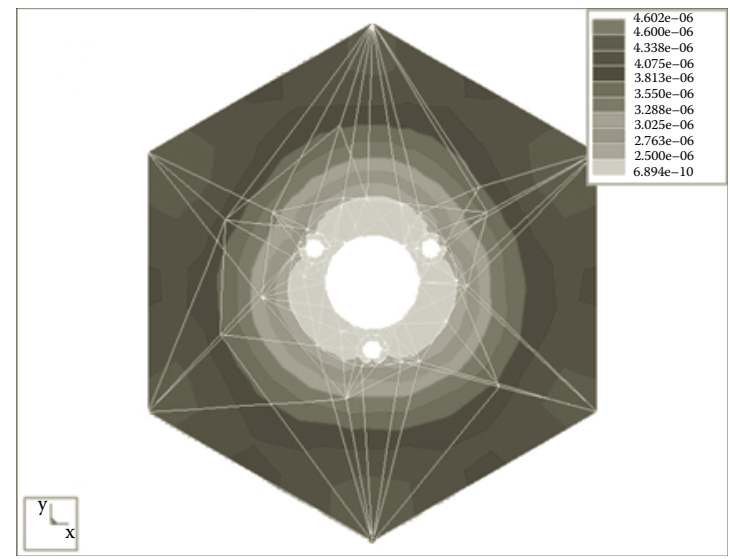

FIGURE 4.7

Finite element analysis of polygon rotating at 30,000 rpm.

In some high-speed applications, distortions in facet flatness can be tolerated. In these cases the structural integrity of the polygonal mirror must be considered. The speed at which the dynamic stress will reach the yield strength (causing permanent distortion and dangerously close to the breaking speed) is found using the formula below. ${ }^{1}$

$$
B=\sqrt{\frac{S}{(7.1 e-6) w\left[(3+m) R^{2}+(1-m) r^{2}\right]}}
$$

where $B=$ maximum safe speed $(\mathrm{rpm}), S=$ yield strength $\left(\mathrm{lb} / \mathrm{in}^{2}\right), w=$ weight of material $\left(\mathrm{lb} / \mathrm{in}^{3}\right), R=$ outer tip radius (in), $r=$ inner bore radius (in), and $m=$ Poisson's ratio. This formula does not have a margin of safety, so it would be wise to consider this and back off the results by an appropriate margin.

\subsection{POLYGONAL MIRROR FABRICATION TECHNIQUES}

Aluminum is the most common substrate for the fabrication of polygonal mirrors. There are two techniques for fabricating aluminum polygons that are widely used. These techniques are conventional polishing and single point diamond turning. Each technique has its advantages and the application will usually dictate the technique used.

\subsubsection{Conventional Polishing}

Conventional polishing in this context is pitch lapping in much the same manner as glass lenses and prisms are polished. A polishing tool is covered with a layer of pitch and a polishing compound is used that is a slurry composed of iron oxide and water. The pitch lap rubs against the optic using the polishing compound to remove material. Pitch lapping can be used to produce high-quality surfaces on a number of materials. Unfortunately, aluminum is not one of them. The aluminum surface is too susceptible to scratches during the 
polishing process. New techniques have been developed, but these rely on minimal abrasive mixtures and therefore material removal rates that are too slow to be cost effective.

Because one cannot polish the aluminum directly, a plating must be applied prior to polishing. Electroless nickel is the most common plating applied. This combination provides the low cost and ease of machining of the aluminum substrate with the superior polishing properties and durability of nickel. The mirror facets are polished individually, blocked up in a surround as shown in Figure 4.8. If the polygonal mirror is regular then a stack of polygons can be polished in one setup.

\subsubsection{Single Point Diamond Turning}

Single point diamond machining is a process of material removal using a finely sharpened single-crystal diamond-cutting tool. Diamond machining centers are available in the form of lathes and mills. The use of ultra-precise air-bearing spindles and hydrostatic table ways, coupled with vibration isolating mounting pads, enables machining to optical quality surface specifications. Figure 4.9 shows a diamond machining center with a polygon in process.

Diamond machining has proven to be an efficient process for generating optical surfaces since it can be automated and the process time is a small fraction of the time required for conventional polishing. The diamond machined mirror is typically fabricated from aluminum, but satisfactory results have been obtained on other substrates. The diamond machined mirror face appears to be a perfect mirror, but upon close inspection the residual tool marks on the surface are apparent. These tool marks create a grating pattern on the surface. This grating pattern can increase the scatter coming from the surface, particularly at wavelengths below $500 \mathrm{~nm}$.

\subsubsection{Polishing versus Diamond Turning}

Diamond turned aluminum scan mirrors are by far used in the highest volumes. This is due to the low manufacturing cost and good performance characteristics. Polished mirrors,

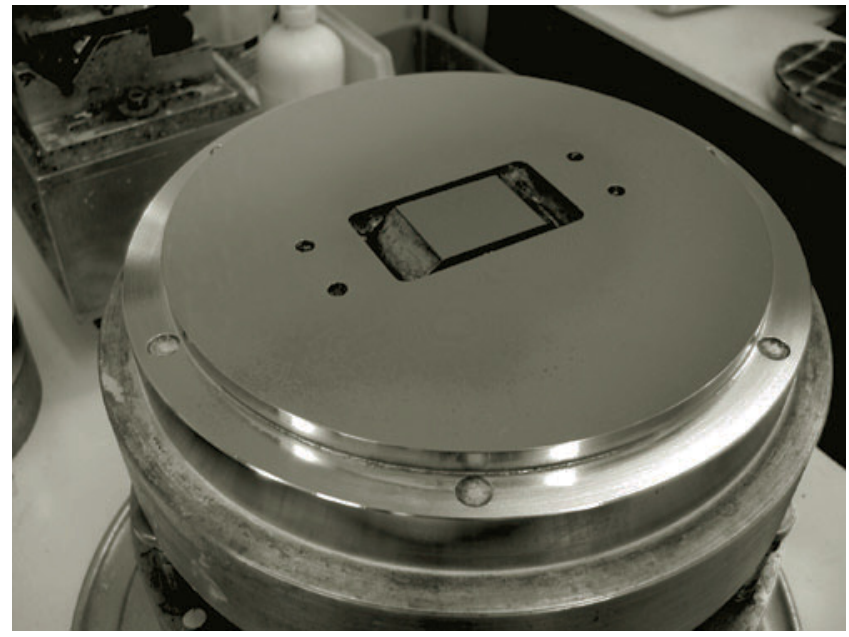

FIGURE 4.8

Conventional polishing of polygonal mirrors. 


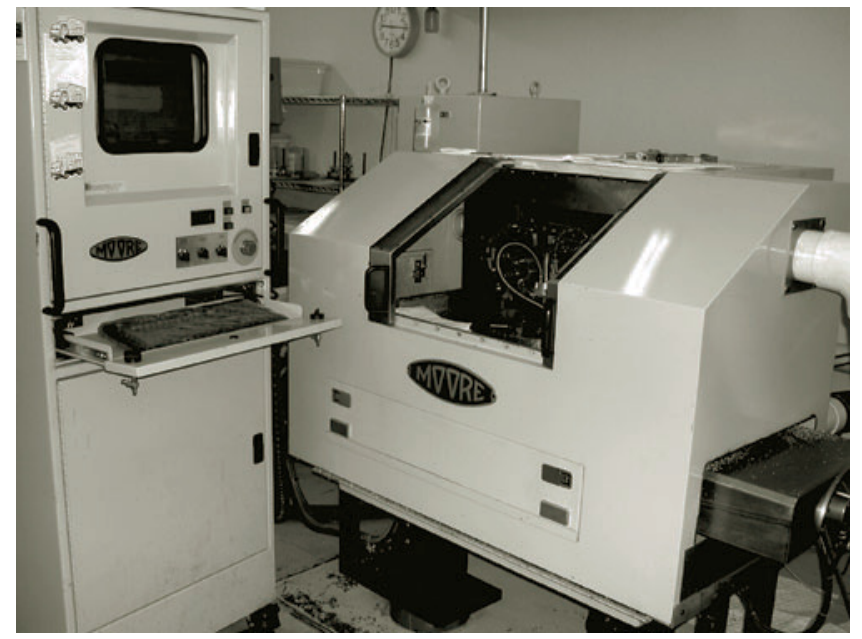

FIGURE 4.9

Diamond turning center.

however, have found a niche where they outperform diamond turned mirrors and justify the higher cost. These applications tend to be very scatter sensitive, such as writing on film. A polished mirror can approach surface roughness levels of $10 \AA$ rms whereas diamond turned mirrors are limited to roughness levels of about $40 \AA$ rms. Short-wavelength applications may also require the lower scatter of a polished mirror surface. Applications below $400 \mathrm{~nm}$ frequently need the lower scatter level of polished mirrors and the scatter can be a problem in applications up to about $500 \mathrm{~nm}$.

There is also a difference in the type of scatter produced by the two surface types. Polished surfaces tend to produce lambertian scatter pattern which has a large wide-angle component whereas diamond turned surfaces produce a large percentage of low angle scatter. The low angle scatter travels along a path close to the specular reflection so it can be difficult to mask out in an optical system.

\subsection{POLYGON SPECIFICATIONS}

In addition to selecting the type of polygon, the material to use, and the fabrication technique, several mechanical specifications need to be established. In a perfect world the polygon would have exactly the dimensions and angles that we specify on a print. Real-world manufacturing limitations cause us to have to add in a practical set of tolerances on the polygon and evaluate how these imperfections would affect system performance. Some of the items that need to be specified on a polygonal mirror include:

1. Facet-to-facet angle variance

2. Pyramidal error

3. Facet-to-axis variance (total and adjacent facet) 
4. Facet radius:

i. nominal tolerance,

ii. variation of all facets tolerance;

5. Surface figure (composed of power and irregularity)

6. Surface quality and scatter

\subsubsection{Facet-to-Facet Angle Variance}

The definition of facet-to-facet angle variance $(\Delta)$ is the variation in the angle between the normals $(\nabla)$ of the adjacent facets on the polygon (Figure 4.10). This variation in angle causes timing errors from one facet to the next as the polygon rotates. Typical values for this angle range from \pm 5 arc $s$ to \pm 30 arc s. Most scanning systems are not sensitive to errors in this range because of the use of start of scan sensors and/or encoders.

This, as well as all other angular tolerances, is a mechanical measure. The system designer needs to ensure that everyone is discussing these errors in mechanical rather than optical terms since there is a factor of two involved between the mechanical tolerances and the optical effects.

\subsubsection{Pyramidal Error}

Pyramidal error is defined as the average variation $(\Omega)$ from the desired angle between the facet and the mirror datum (Figure 4.11). This variation results in a pointing error of the output beam and can also cause scan line bow. Typical values for this specification are \pm 1 arc minute (mechanical).

\subsubsection{Facet-to-Axis Variance}

This is defined as the total variation of the pyramidal error from all the facets within one polygon (Figure 4.12). This is a critical specification for the mirror and contributes to a scanner specification of dynamic track, discussed later. Typical values for this specification range from 2 arc $s$ to 60 arc $s$ (mechanical).

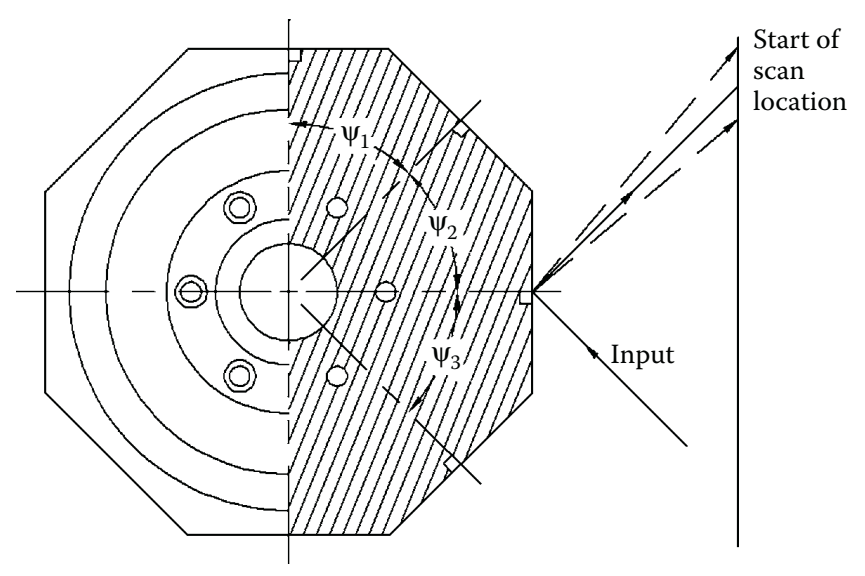

FIGURE 4.10

Facet-to-facet angle variance. 


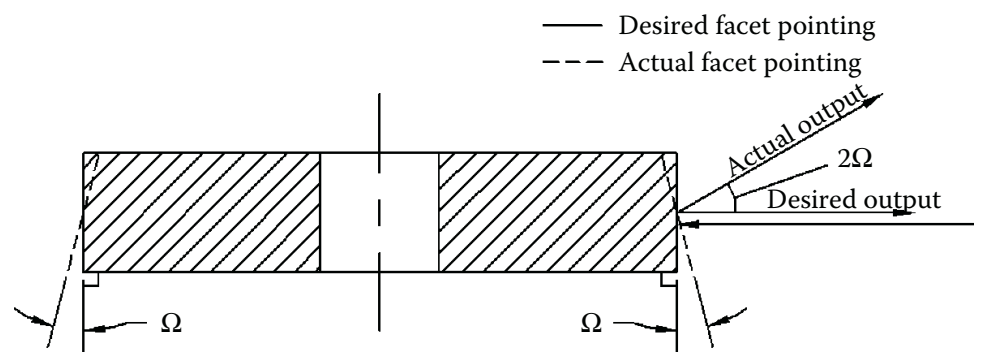

FIGURE 4.11

Pyramidal error.

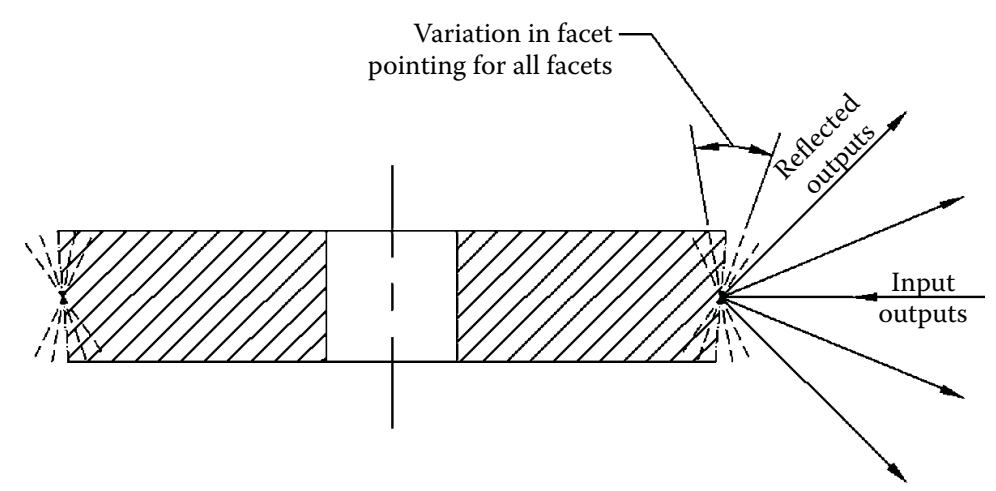

FIGURE 4.12

Facet-to-axis variance.

Another parameter related to this is the adjacent facet-to-axis variance. This is defined as the largest step in the pyramidal angle from one facet to the next within a polygon. This is important to control in order to reduce banding artifacts in the final system. Typical values for this specification are in the range of 1-30 arc s (mechanical).

Optical scanning systems may employ correction devices that allow this value to be reduced. System resolution plays a large part in determining the actual value required. Film writing applications tend to have the tightest requirements and passive reading systems tend to have the loosest requirements.

\subsubsection{Facet Radius}

The facet radius (referred to as facet height by some manufacturers) is the distance from the center of the polygon to the facet. The variation in this radius within the polygon and the tolerance on the average radius are important to specify. The average facet radius is important because it locates the facet in the optical system. The variation of this radius within a polygon causes errors in the focal plane location from one facet to the next. It also causes velocity variations within the scan line, which are usually small and show up as jitter errors. Typical values for these parameters are \pm 60 microns for the facet radius average position and \pm 25 microns for the facet radius variation within a polygon. 


\subsubsection{Surface Figure}

Surface figure is the macro shape of the polygon facet and is measured as the deviation from an ideal flat surface. The flatness of polygon facets will have an impact both on the aberrations in the beam as well as the pointing of the beam. The aberrations can affect the final focused spot size in the scan system. The pointing error results in velocity variations across the scan.

Several factors influence the flatness of polygon facets:

1. Initial fabrication tolerances

2. Distortion due to mounting stresses

3. Distortion due to forces induced when rotating at high speeds

4. Distortion due to long-term stress relief

Interferometers are commonly used to measure static flatness. The flatness is specified in wavelengths, $\lambda$, (or fractions thereof) of light. A typical flatness specification is: $\lambda / 8$ at $633 \mathrm{~nm}$. Departure from flatness can have a variety of forms, depending on how the surface was fabricated. For example, conventionally polished mirror surfaces tend to depart from flat in a regular spherical form, either convex or concave. Diamond machined surfaces usually depart from flat in a regular cylindrical form, either convex or concave. A polygon will typically have two specifications related to flatness, a surface power specification and irregularity. The irregularity is defined as the deviation from a best-fit sphere. Another common way of specifying the optical surface is in terms of power and pv-power (peak to valley error minus power), which separate the regular and irregular shapes. Most polygons used in printing applications are specified in the $\lambda / 8$ to $\lambda / 10$ range at the wavelength of interest.

\subsubsection{Surface Quality and Scatter}

Ideally a reflective optical surface will reflect all of the incident light without introducing any scattered components. In reality an optical surface has multiple defects of various sizes. The U.S. military developed a scratch and dig specification for surface defects, which is included in MIL-PRF-13830B and is in broad use within the optics industry. This method of quality determination involves close examination of a surface and identifying a scratch and dig level in a given unit area. A typical high-quality conventionally polished polygon will have a quality level of 40-20 scratch and dig.

Machined optical surfaces on the other hand, are made up of a precise regular pattern of machine tool marks, which are sufficiently high in frequency and low in height errors as to behave as a plane mirror at most visible and infrared wavelengths. The scratch and dig specification must be supplemented with an additional measure of surface quality here. A more representative definition for the overall surface quality is the rms surface roughness. The rms surface roughness can be measured directly by mechanical or optical profilometry means or indirectly by measuring the scatter from the surface.

A special test system is required to measure the scatter from the surface and correlate this to an rms roughness value. Different tests must be used for the measurement of scatter of diamond turned and conventionally polished mirrors. Diamond turned surfaces produce a significant fraction of scattered energy in a narrow cone around the reflected beam. Conventionally polished mirrors have the majority of their scattered energy in a cone significantly greater than the divergence angle of the reflected beam. 
Conventionally polished mirrors can be tested using an integrating sphere that gathers a wide cone angle (Figure 4.13). Scattered light in the cone of $4-180^{\circ}$ is gathered with this test method. The diamond turned mirrors can be tested using a combination of the integrating sphere and a near angle test (Figure 4.14). Scattered light in the cone of $0.4-4^{\circ}$ is gathered using this test.

A correlation has been developed between the rms surface roughness and the total integrated scattered incident light: ${ }^{2}$

$$
\text { rms roughness }=\frac{1 \sqrt{\ln (1-T I S)}}{4 p}
$$

where TIS is the total integrated scatter.

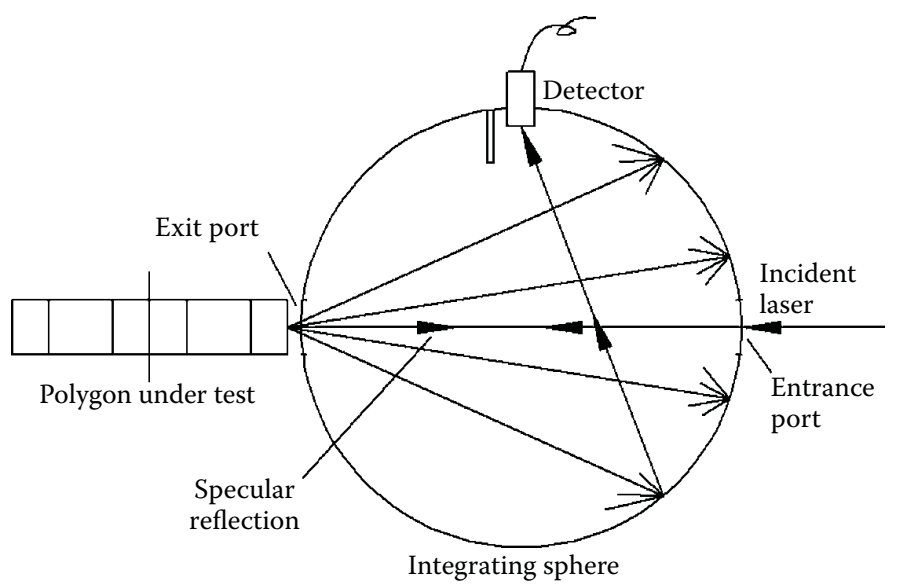

FIGURE 4.13

Test for wide angle scatter.

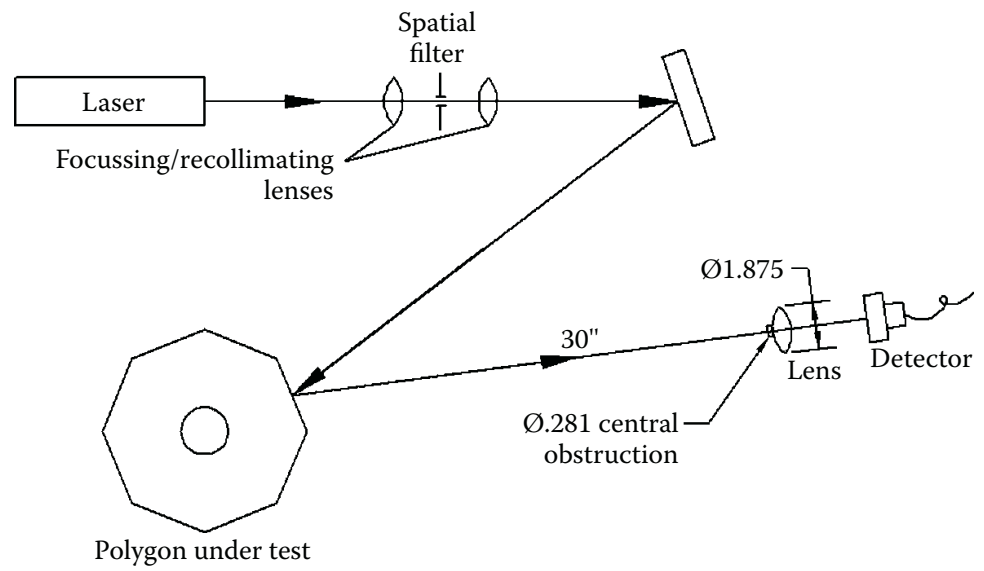

FIGURE 4.14

Test for near angle scatter. 
The combination of scratch and dig along with rms surface roughness provide a good description of the surface structure higher in frequency than surface figure.

\subsection{THIN FILM COATINGS}

There are two major functions of optical coatings on polygons: to improve the reflectance of the surface and/or to improve durability. In the case of diamond machined polygons, the substrate is usually aluminum (in itself a good reflector over most of the visible spectrum). This aluminum surface is too soft without a coating. It is easily scratched during even a light cleaning so one solution is to apply a thin layer of silicon monoxide, a dielectric material, as a surface protector. The optical thickness is usually about one-half wavelength at the wavelength of interest. This material is more durable than the base aluminum and can be readily cleaned. The coating just described is typically referred to as a protected aluminum coating. This coating has a reflectivity of $>88 \%$ across the $450-650 \mathrm{~nm}$ range.

The protected aluminum coating is fine for many applications and because of its simplicity is relatively inexpensive. Many applications, however, require enhancement coatings due to needs for higher reflectivity performance at various wavelengths.

The first layer deposited in most applications is a binding layer, then a metal, such as aluminum, silver, or gold. The layer or layers above the metal are composed of dielectric materials. The metal is selected based on the wavelengths of interest. As mentioned earlier, aluminum is a good choice in the visible region. It is also selected for ultraviolet applications since its reflectivity can be enhanced in this region with a dielectric stack. Gold is often selected as the base metal in applications above $600 \mathrm{~nm}$. It has good reflectivity at $600 \mathrm{~nm}(90 \%)$ and very good reflectivity from 1 micron, out past 10.6 microns ( $>98 \%)$.

Silver exhibits very desirable reflectance characteristics over a broad spectrum and is frequently considered as a material for polygon coating. In practice, however, it is frequently a disappointing choice for the long term. The slightest pinhole (or minute scratch from cleaning) will expose the silver to reactive contaminants from the atmosphere, which over time (several days or weeks) will diffuse into the silver, producing an expanding blemish. Aluminum, which initially exhibits somewhat lower values of reflectance than silver, is far superior in terms of durability.

Common dielectric materials used to protect the surface and enhance reflectivity are silicon monoxide, silicon dioxide, hafnium oxide, and titanium dioxide. A quarter wave stack combining high refractive index and low refractive index materials is used to enhance the reflectivity in the wavelength region of interest. The term quarter wave stack refers to an alternating series of high and low index materials that are one-quarter of an optical wavelength thick at the wavelength of interest. The design of this quarter wave stack can be tailored to raise the reflectivity of the base metal significantly in various regions of interest. Most companies will offer a variety of standard reflectivity enhancing coatings for different wavelengths.

Thin film coatings are applied in vacuum deposition chambers such as the one shown in Figure 4.15. The tooling to coat a polygon is specialized because the polygon has optical surfaces around its periphery. The polygons are stacked onto coating arbors that are placed in the chamber above the evaporant sources. The arbors are rotated with a drive mechanism during the deposition process at a constant rate so that all facets will see the same thickness of coating material. The rotation rate must be fast enough that the time for 


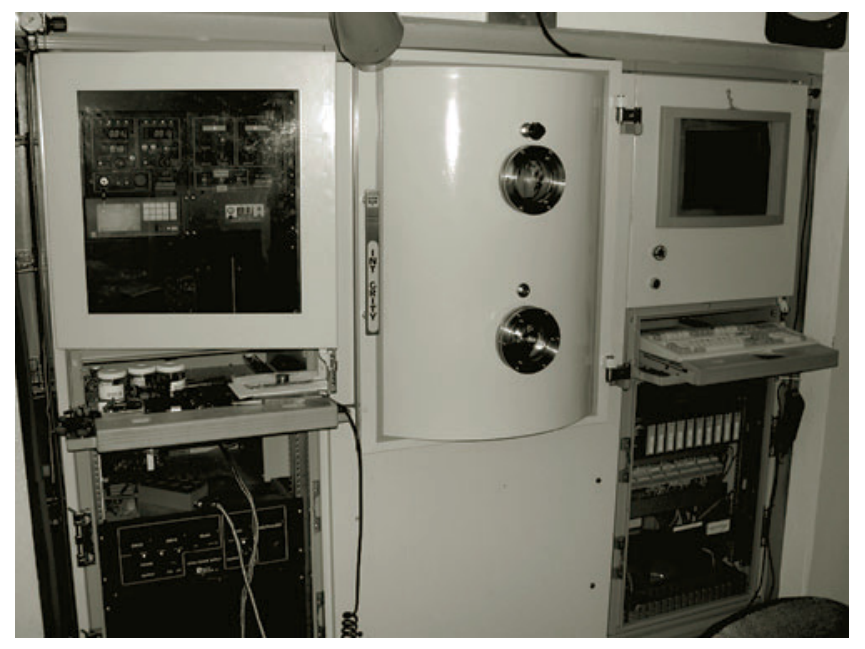

FIGURE 4.15

Thin film deposition chamber.

one revolution is a small fraction of the deposition time for a single layer. Otherwise there will be significant variation around the polygon depending on when the shutter is opened and closed.

Reflectance uniformity both within a facet and facet-to-facet is an important polygon specification. The reflectance uniformity can impact the accuracy of written or read images. In practice, the reflectance uniformity of rather large polygon facets (e.g., a few inches square) is more difficult to achieve than if the facets are small (e.g., $\frac{1}{2}$ in square). This has to do with the consistency of the cleaning of the surface prior to coating and the variations in deposition rates with location and time within the coating chamber.

Aluminum polygons cannot be heated up to high temperatures during a coating run as one would typically do for good adhesion and layer density. The shape of the polygons makes them susceptible to slight stress changes during this heating cycle. This results in changes to the facet flatness. The coating process should be designed to keep the polygons below a temperature of $225^{\circ} \mathrm{C}$ to maintain the flatness.

Crucial to all of the desired characteristics of the coating of a polygon is its cleanliness prior to coating. Irrespective of the fabrication methods, polygons will be handled prior to coating during the inspection processes, transport, and installation into the coating chamber. The polygon must be cleaned thoroughly to remove foreign material that will degrade surface quality, prevent good coating adhesion, or outgas in the coating system.

Several tools are available to measure the optical performance of the coating. Common measuring tools to determine reflectance are spectrophotometers and laser reflectometers. Spectrophotometers are used to provide information on the reflectance versus wavelength. The majority of spectrophotometers with a reflectance measuring attachment are limited to small sample sizes on the order of 1 to 2 in in diameter. This fact usually precludes measuring the polygon itself. A witness sample is coated at the same time as the polygon and can be used to represent the actual part performance. This can be a reliable method of ascertaining the performance of the polygon as long as the witness sample has a similar surface preparation and quality level to the polygon. This means that diamond turned witness samples should be used with diamond turned polygons and polished witness samples used with polished mirrors. 
Laser reflectometers compare the reflected beam to the incident beam at a specific wavelength and can be designed to test over a range of angles with either S or P polarization. Reflectometers are useful for determining performance at one specific wavelength but cannot provide broadband information.

\subsection{MOTORS AND BEARING SYSTEMS}

The polygonal mirror requires a bearing system and a drive mechanism to turn it into a functional scanner. Drive mechanisms include pneumatic, AC hysteresis synchronous, and brushless DC. Bearing systems used in most applications are ball bearing, aerostatic air bearings, or aerodynamic air bearings.

\subsubsection{Pneumatic Drives}

Much of today's scan mirror technology has evolved from the development of ultrahigh-speed polygon/turbine motors for the high-speed photography industry. Compressed air turbines continue to offer an attractive method of rotating a polygonal mirror at speeds beyond the capability of electric motors. The advantages of turbine drives are:

1. Substantial horsepower can be delivered to the scan mirror to produce rapid acceleration and very high speed (up to 1,000,000 rpm).

2. Compact in size and low in weight in proportion to delivered power.

3. Can be equipped with shaft seals so that the scan mirror can be used in a partial vacuum.

The disadvantages of turbine drives are:

1. Require a compressed air source

2. Asynchronous devices

3. Relatively high in cost

4. Relatively short total running life

Pneumatic drives are only recommended for short duty cycles and where ultra-high speed is essential.

\subsubsection{Hysteresis Synchronous Motors}

The rotor of a hysteresis synchronous motor is usually fabricated from a single piece of hardened steel selected out of a group (predominantly alloyed with cobalt) that exhibits substantial hysteresis loss. This resistance to the movement of magnetic flux in the material imparts torque to a rotor out of sync with the drive current. This torque is responsible for the motor's ability to start rotation. When the rotor approaches the speed of the stator flux, it becomes permanently magnetized and "locks in" to synchronism with the drive. If the motor is turned off and restarted, the stator flux demagnetizes the rotor and hysteresis 
takes over again. The synchronous mode of operation is more efficient than the hysteresis or startup mode, and in many systems a sync detector is used to reduce drive current after the motor is locked in to save energy and reduce heating.

AC hysteresis synchronous motors exhibit a characteristic called phase jitter (hunting). The rotors behave as though they were coupled to the drive waveform by a spring. Within synchronism the rotor springs forward and back in phase at a rate determined by the spring rate (flux density) and the torque/inertia ratio of the system. Typically, the frequency of this phase jitter is in the range of $0.5-3 \mathrm{~Hz}$, at an amplitude of a few degrees $\left(1-6^{\circ}\right.$ peak to peak). Under perfect conditions this jitter damps to zero values of amplitude. However, perfection is seldom seen and continual recurrence of jitter may be expected, caused by electrical transients on the input, mechanical shock to the assembly, variable resistance torque of the motor bearings, and so on. For many systems the $0.5-0.01 \%$ velocity error contribution of phase jitter is acceptably small. If this is not the case then a feedback loop is needed to reduce this level.

\subsubsection{Brushless DC Motors}

Brushless DC motors are by far the most common motor used to drive polygonal scanners. These motors use a permanent motor magnet and a stator that supplies the varying magnetic force. Motor magnets are composed of various materials including neodymium and ferrite depending on the application. Stators can be iron-based or ironless, with or without teeth. The number of magnetic poles is usually determined by the operating speed. Lowspeed motors tend to have higher pole counts $(8-12)$ while higher speed motors $(>10,000$ $\mathrm{rpm}$ ) tend to have lower pole counts (4-6). The reason for the large number of poles at low speed is to achieve smoother rotation. At higher speeds this is not required and the lower pole count motors have less losses because the stator flux speed is lower.

Brushless motors do not have the hunting problem associated with AC hysteresis synchronous motors. The motor controls used to drive these motors can hold a tighter control loop. These motors can exhibit more high-frequency variations due to the torque available to rapidly change speed. This high-frequency velocity change is referred to as jitter. The amount of jitter is related to the rotor inertia and the number of feedback pulses per revolution. At higher speeds the inertia smoothes out the rotation and limits the amount of jitter. At lower speeds the number of feedback pulses helps keep the control loop errors small and therefore less velocity jitter when the motor has a correction torque applied. Hall effect devices are used at higher speeds to provide magnetic position feedback to the controller. Hall effect devices at lower speeds, where inertia is lower, can induce jitter as the controller chases the positional and triggering errors of the Hall effect devices. At lower speeds an encoder on the rotor may be required to achieve low jitter levels. Even incremental encoders can induce jitter errors due to disc quality, alignment, and component quality.

\subsubsection{Bearing Types}

Polygonal scanners require a bearing support system to allow the rotor to rotate. The most common bearings used in scanners are:

1. Ball bearings

2. Aerostatic air bearings

3. Aerodynamic air bearings 
These three types of bearing systems are discussed in detail in other chapters in this book. Ball bearings are used where possible due to their low cost. Applications requiring speeds less than 20,000 rpm and that can tolerate bearing nonrepeatable errors, both in scan and cross scan, are candidates for ball bearings.

Aerodynamic air bearings have made large inroads in laser scanning since the 1980s. An aerodynamic bearing generates its own air pressure as it rotates. It is commonly designed with two close-fitting cylinders for the radial bearing. The axial bearing can be either an air thrust bearing or magnetic bearing. These systems have many advantages over both conventional ball bearing systems and aerostatic air bearings. The speed range for aerodynamic bearings is from approx. $4000 \mathrm{rpm}$ to over 100,000 rpm. These bearings are only slightly more costly than an equivalent ball bearing system. There is no wear while operating and they require no external pressure support equipment. These bearings have been developed to withstand over 20,000 start/stop cycles. Aerodynamic bearings do have some limitations that limit their application. They are not well suited to dirty environments. Many designs exchange outside air frequently during operation, thereby ingesting the outside debris. Most designs cannot withstand high shock loads because the bearing stiffness is limited. The mass of the optic is limited in many applications due to both the lack of support and the need to withstand constant starting and stopping. Additional mass causes added wear to the bearing during startup and shutdown.

Aerostatic air bearings provide the ultimate in performance at a high cost. An aerostatic bearing uses pressurized air and closely spaced axial and radial bearing surfaces to float the rotor. When pressurized, the bearing has no contacting parts, resulting in extremely long life. These bearings are very stiff and have wobble errors less than 1 arc s. They are capable of supporting heavy loads and do not suffer from wear at startup and shutdown. They do require external components to supply the pressure to the bearing. This increases system complexity as well as cost.

\subsection{SCANNER SPECIFICATIONS}

Once the polygon, motor, and bearing system have been decided on, the packaging of the assembly becomes the next concern. One of the key elements in attaining high scanner performance is the mounting of the scan mirror to the rotating spindle.

To preserve the facet flatness achieved during initial polygon fabrication, it is necessary to fasten the polygon to its drive spindle with care, particularly if $\lambda / 8$ or better flatness is required. The interface between the mirror and the rotor must not induce stress in the mirror that is translated out to the facets.

A typical mounting scheme is shown in Figure 4.16. In this case the datum surface of the polygon and the locating annulus of the mounting hub are lapped to optical quality so that when the two are firmly held together, distortions are minimized.

Equally important to the accurate mounting of mirror datum and rotor hub surfaces is cleanliness at assembly and the appropriate torque levels of the fastening screws. Polygons can be attached in the manner described in low- and medium-speed applications. When tip velocities approach $76 \mathrm{~m} / \mathrm{s}$, other methods of mounting need to be considered.

In many applications the facets can be allowed to distort as long as they all change by the same amount. A symmetrical mounting method with screws aligned with every apex will work in this type of application. Other applications cannot stand significant shape change 
on the facets and require a true radially symmetric mounting method such as clamping. Clamping has been used successfully but this also requires a radial attachment means that may consist of an elastic material or aluminum shaped to have a spring force.

Once the polygonal mirror is integrated with the motor and the bearing system it can be referred to as a polygonal scanner. The scanner assembly is defined by performance specifications that include:

1. Dynamic track

2. Jitter

3. Speed stability

4. Balance

5. Perpendicularity

6. Time to sync

\subsubsection{Dynamic Track}

Dynamic track is defined as the total mechanical angular variation of the facets perpendicular to the scanning direction. This is illustrated in Figure 4.17. An optical beam illuminating a polygon with a dynamic track of 10 arc $s$ will have a scan envelope from all the

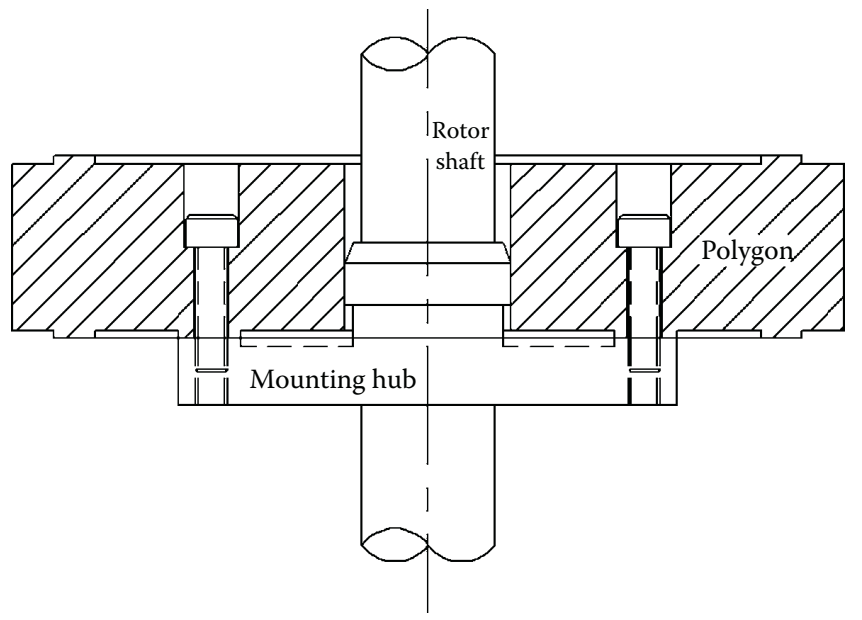

FIGURE 4.16

Mirror/rotor interface.

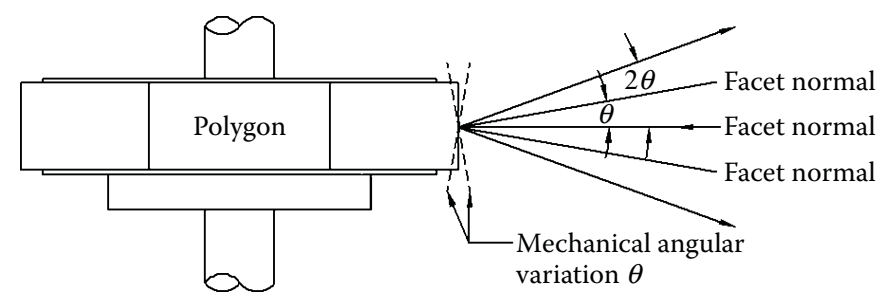

FIGURE 4.17

Dynamic track errors. 
facets of 20 arc s perpendicular to the scan direction. This is caused by an angle doubling effect on reflection from the rotating mirror.

There are four significant contributors to dynamic track error. The first is the polygon itself, which has a variation in the angle of each facet and can have a residual pyramidal (squareness) error. The second contribution comes from the mounting of the polygon to the rotating shaft. If the polygon is not perfectly perpendicular to the rotating shaft, then the facets will change their pointing in a sinusoidal manner with a period of one revolution. These first two contributions are fixed and repeatable. The third contribution is a random nonrepeatable error caused by the bearing support system. The random component of the dynamic track error will be 1-2 arc $\mathrm{s}$ for a ball bearing assembly and less than 1 arc $\mathrm{s}$ for air bearing assemblies. The fourth contribution, which is a repeatable error, comes from the grinding operation performed on the mounting hub of the spindle. This hub needs to be perpendicular to the rotation axis of the spindle and typically a few arc s error is present due to the grinding tolerance.

The repeatable component of dynamic track (which tends to be larger) will show up in a laser writing system as a banding artifact. The line spacing will not be uniform and will repeat the pattern on each revolution of the polygon. Dynamic track errors can be reduced, if needed, through either active or passive correction means. These are methods discussed in Section 4.12.1.

\subsubsection{Jitter and Speed Stability}

Velocity errors from a polygonal scanner are important to minimize because they affect the pixel placement in a writing application and the receiving angle in a reading application. Velocity errors have both repeatable and nonrepeatable components. The repeatable components are easier to deal with than the nonrepeatable errors.

Specifications for velocity errors are broken into both high-frequency (jitter) and lowfrequency (speed stability) components. The high-frequency components range from pixel-to-pixel to once per revolution. The low-frequency components are over multiple revolutions. There are many elements of the scanner system that contribute to either jitter or speed stability errors. These contributing elements are shown in Table 4.1.

This is a long, but certainly not exhaustive, list of causative elements contributing or potentially contributing to velocity errors. It becomes obvious that the entire scanner optical system is involved and influences the speed stability measurement and result.

\subsubsection{Balance}

Polygonal scanners are rotational devices that can operate at high speeds. As such, they need to be properly balanced to reduce the amount of imbalance forces generated during operation. This includes compensating for both static and dynamic imbalance. This requires that a two-plane balancing system be used. In a two-plane balancing system sensors are located at two separated planes where correction weights are to be applied. The sensors record the magnitude and phase of the imbalance.

Various methods of either adding or removing weight are used to balance scanners. The most common techniques are:

1. Drill balancing

2. Epoxy balancing

3. Screw balancing

4. Grind balancing 
TABLE 4.1

Elements Contributing to Jitter or Speed Stability

Errors

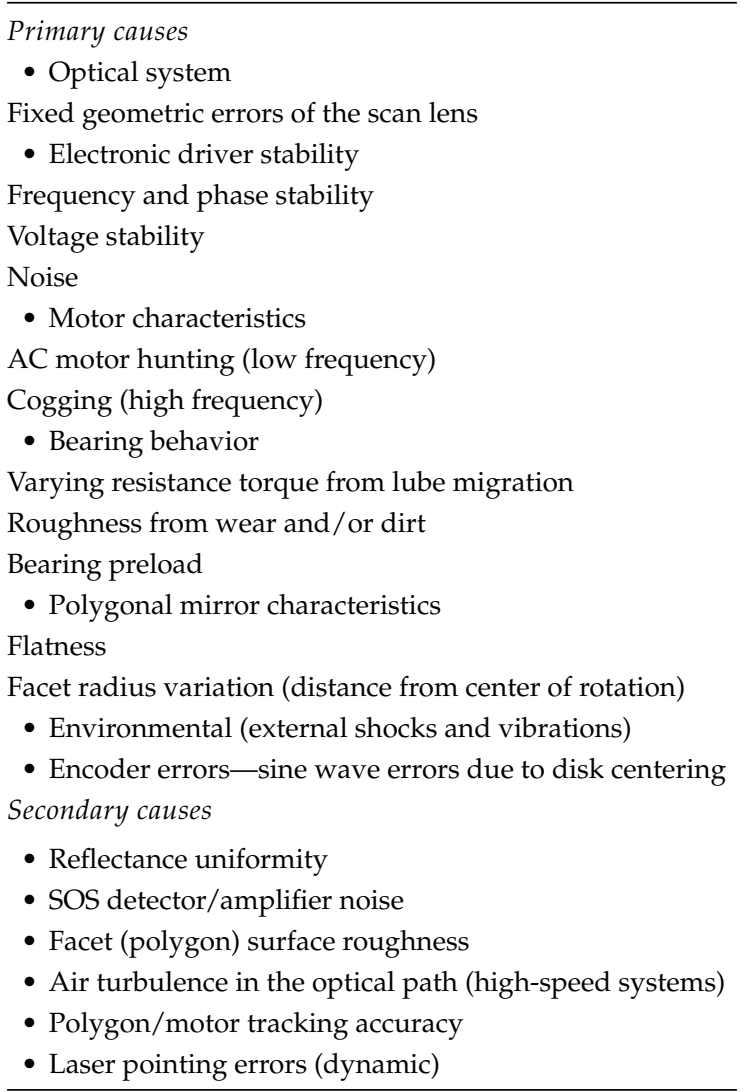

The preferred approach for high-speed operation is either grinding or drilling to remove material. The addition of material always brings risk of improper attachment and slinging of bonding agents.

Unbalance is typically measured in $\mathrm{mg} \mathrm{mm}$, a mass multiplied by the distance from the rotational axis. An unbalance of $100 \mathrm{mg} \mathrm{mm}$, for example, indicates one side of the rotor has excess mass equivalent to $100 \mathrm{mg}$ at a 1-mm radius. Typical values for small highspeed scanners range from 10 to $100 \mathrm{mg} \mathrm{mm}$. The impact of unbalance on a scanner is vibration. This vibration can be measured and from this the actual scanner unbalance can be calculated.

\subsubsection{Perpendicularity}

Another important scanner parameter is the perpendicularity of the rotation axis to the mounting datum. This is important to ensure proper pointing of the beam after reflection from the polygon and to minimize the bow that can be created by striking the polygon out of the rotation plane. A typical specification for perpendicularity is 3 to 5 arc minutes. 


\subsubsection{Time to Synchronization}

The time that it takes for the scanner to reach operating speed from a stopped condition can be important in some applications. This is a function of the motor/winding and the available current as well as the rotor inertia and the windage that must be overcome as the scanner approaches operating speed. Typical values range from 3 to $60 \mathrm{~s}$.

\subsection{SCANNER COST DRIVERS}

Polygonal scanners can range from low-cost, easy-to-manufacture units, to high-cost stateof-the-art devices. It is important when designing a scan system to understand the cost drivers. One should try to minimize the overall cost through system level trade-offs. The scanner assembly has many cost drivers including:

1. Polygon shape

2. Number of facets

3. Fabrication method, conventionally polished or diamond turned

4. Optical specifications including surface figure, surface roughness, and scratch/dig

5. Coating requirements

6. Polygon size

7. Type of bearing system

8. Speed

9. Velocity stability

10. Dynamic track specification

In an earlier section the various shapes of polygons were discussed. In order to reduce costs it is advisable when possible to select either a regular polygon or a monogon. The other polygon shapes have cost penalties that may or may not be justified based on the application. While polygons can be manufactured with any number of facets, fewer facets result in lower cost. This is not a large cost component in a diamond turned mirror but has a large impact on the cost of a polished mirror.

The selection of diamond turned or polished mirror has a major impact on scanner cost. Diamond turned mirrors are the lowest cost and have surface roughness values greater than $40 \AA$ rms. Conventionally polished mirrors are more costly but can bring the surface roughness down to $10 \AA \mathrm{rms}$. All but the most scatter-sensitive short-wavelength systems can use diamond turned mirrors.

The optical specification of surface figure can also have a large influence on cost. Optical surface figure values of $\lambda / 4$ per inch at $633 \mathrm{~nm}$ are common but surface figure values down to $\lambda / 20$ can be achieved at additional cost. A scratch/dig specification of $80 / 50$ is a typical standard, but specifications down to $10 / 5$ can be achieved at significantly higher cost.

The optical coating chosen for the polygon can have a minor impact on the cost. The lowest cost option is a simple aluminum coating with a silicon monoxide overcoat. As the reflectivity specifications get higher, more dielectric layers are needed to enhance the reflectivity, which can increase chamber time and therefore costs. Gold is another 
expensive coating option for the infrared. The inherent high reflectivity of gold across the IR spectrum is often worth the cost of the material.

Bearing selection can have a significant effect on cost. In the speed range of 500-4000 $\mathrm{rpm}$, the choice is between ball bearings and aerostatic air bearings. Ball bearing scanners are relatively low in cost and are the appropriate solution for many applications, but are susceptible to damage, generate many vibration frequencies, and can create motor speed instability. Aerostatic scanners are costly and require support equipment, but offer the ultimate in scanning performance.

The bearing choice in the speed range of 4000-20,000 rpm includes ball bearings, aerodynamic air bearings, and aerostatic air bearings. The selection is based on cost and performance criteria such as velocity stability and dynamic track. Above 20,000 rpm, aerodynamic air bearings are usually the best solution. These bearings are relatively low in cost and have long life operating at this speed. Ball bearings start to have life issues above 20,000 rpm and aerostatic bearings usually are not cost effective.

Velocity stability standard specifications are a function of speed and mirror load. If speeds are too low or mirror loads too small then an encoder is required to achieve tight velocity stability. Velocity stability in this context is a measurement of the variation in the time for a beam reflected from the same facet of a scanner to cross two stationary detectors in an image plane over 500-1000 revolutions. Scanners operating faster than $4000 \mathrm{rpm}$ can easily achieve $0.02 \%$ velocity stability. On most units this can be improved upon down to $0.002 \%$ at additional cost. Below $4000 \mathrm{rpm}$ the mirror load becomes very important. The lighter the mirror and slower the speed, the more difficult it is to achieve tight velocity stability.

A final significant cost driver is the track specification placed on the assembly. Mechanical track values of 45 arc $s$ results in low-cost assemblies, but specifications as tight as 1 arc $\mathrm{s}$ can be achieved by some vendors at much higher costs. This specification is a serious cost driver, so it is recommended that you review your actual needs carefully to obtain the most cost-effective design.

\subsection{SYSTEM DESIGN CONSIDERATIONS}

Laser scanning systems based on polygon technology can take on a variety of forms. Systems can range from very simple to extremely complex based on the performance level required. The first system consideration is whether it will be a reading or writing system. Writing systems tend to have much tighter performance requirements than reading systems. This is due to the fact that writing system errors tend to be visible, whereas the same level of error in a reading system will not typically be great enough to impact data integrity. A reading system, however, has the additional complexity of collecting the scattered light back from the target.

Reading systems will either use an external collection system that is separate from the scan system or an internal collection system where the scattered light passes back through the scan system and is derotated by the polygonal scanner. The internal collection system places increased demands on the scan system by requiring less backscattered light and reduced ghost images. For laser radar systems, one often has a scanning system for transmitting the laser, and a separate receiver, with a synchronized scanner to avoid this problem. This, however, is a very expensive solution. Another approach taken with laser 
radar is to increase the facet width and separate the transmission and receive apertures. Care must be taken in the design to ensure that the receiver instantaneous field of view encompasses the transmitter output over the distance range desired. This topic is covered in greater detail in the chapter on laser radar later in this book.

Beyond having knowledge of the basic system configuration it is important to develop a thorough list of performance specifications when starting the system design process. A list of key parameters and typical values are shown in Table 4.2.

The list in Table 4.2 covers the majority of specifications that are placed on a scanning system. Some scan systems will require additional specifications based on the unique nature of the writing or reading application.

The optical system used in laser scanners can be separated into two generic types: preobjective and post-objective. Pre-objective is a term used to describe the use of a polygon to deflect a ray bundle, which after deflection is imaged by a lens or curved mirror (Figure 4.18). This method of scanning places the function of focal plane definition on the lens, referred to as a scan lens, rather than on the scanning facet. Several desirable characteristics can be designed into the scan lens when employed in pre-objective scanning. An example is a lens design referred to as F-Theta. An F-Theta lens has the following characteristics:

1. A flat focal plane

2. Uniform spot diameter over the entire scan

3. Linear spot velocity at the scan plane (assuming constant angular velocity of the polygon)

Usually it is desirable to have the scanning spot move with a highly accurate and constant velocity in the scan plane. Polygonal mirror deflectors provide angular velocity stability in the range $0.002 \%-0.05 \%$, depending on the speed and inertia of the scanner. Without the aid of an F-Theta lens, however, the spot velocity variation on a flat focal surface will be

\section{TABLE 4.2}

\begin{tabular}{ll} 
List of Key Parameters & \\
\hline Wavelength & $350-10,600 \mathrm{~nm}$ \\
Number of resolvable points & $100-50,000$ \\
Spot size & $1 \mathrm{micron}-25 \mathrm{~mm}$ \\
Spot size variation across scan & $\leq 5 \%-15 \%$ \\
Scan length & $1 \mathrm{~mm}-2 \mathrm{~m}$ \\
Telecentricity & $0.5-30^{\circ}$ \\
Bow & $\leq 0.001 \%$ of scan line length \\
Scan efficiency & $30 \%-90 \%$ \\
Intensity nonuniformity & $\leq 2 \%$ to $\leq 10 \%$ \\
Pixel placement accuracy & \\
$\bullet$ Jitter & $\leq 0.002 \%$ to $\leq 0.02 \%$ \\
$\bullet$ Cross-scan error & $\leq 1 \%$ to $\leq 25 \%$ of line spacing \\
Scatter & $\leq 0.2 \%$ to $\leq 5 \%$ \\
Data rate & \\
Laser noise levels & \\
Environmental factors and system interfaces &
\end{tabular}




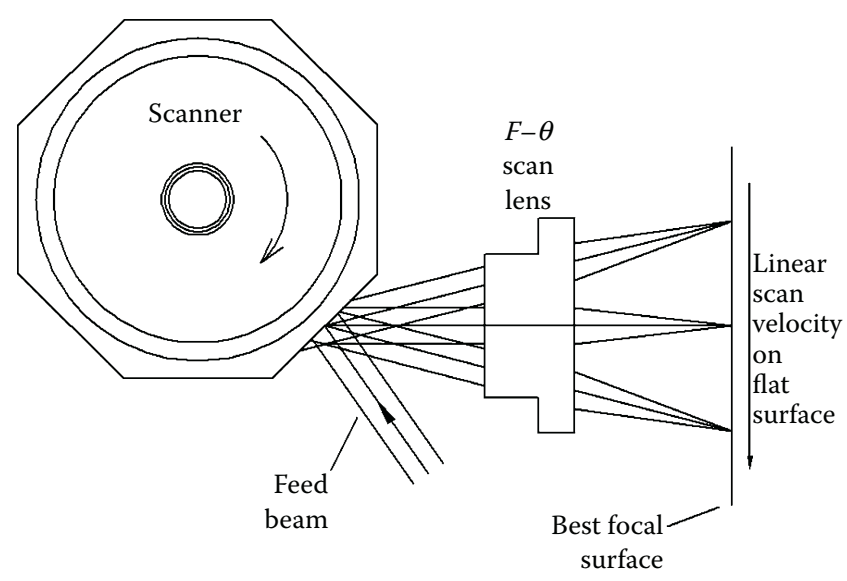

FIGURE 4.18

Pre-objective scanning system.

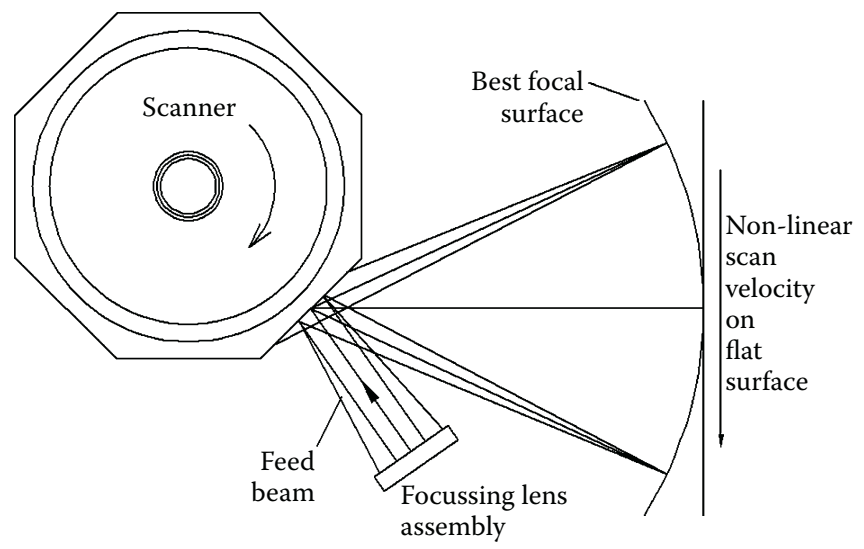

FIGURE 4.19

Post-objective scanning system.

proportional to the tangent of the scan angle, which for systems involving several degrees of scan means several percent variation.

Post-objective scanning is a term used to describe the use of a polygon to deflect a focusing ray bundle over a focal surface (Figure 4.19). This method places the function of focal plane definition on the polygonal mirror, and the imaging (spot forming) lens is a relatively simple component located prior to the polygon.

The focal surface of a post-objective scanner is curved. The center of curvature is the center of the polygon facet. This type of scan system is typically used when the scan plane can be curved to match the focal surface. Otherwise there are problems with spot size and velocity variations across the scan. The most popular system design incorporating postobjective scanning is a drum scanner. A drum scanner uses a monogon mirror, usually at $45^{\circ}$, with the source on the scan axis. As the monogon rotates, the focal surface is generated on the inside of a drum. Film or other flexible medium is located on this drum for image generation. 
Post-objective scanning finds application in very high-resolution systems requiring greater than 25,000 points across the scan. Scanners designed for the prepress industry use this design technique quite often.

Another factor to consider when designing a scan system is the degree of telecentricity required. A system is considered to be telecentric if the output from the scan system strikes the image plane at $90^{\circ}$ for all points across the scan line. A post-objective scanner can be telecentric if the image plane can be curved to intercept the output from the scanner. If a flat image plane is required, a pre-objective scan system will need to have a scan lens that is slightly larger than the scan plane to meet the telecentric requirement. This can drive up the scan lens costs and result in a prohibitively expensive system. Normally, some level of deviation from telecentricity is given in a system specification.

In a writing application a decision as to how to use the available polygon facet is needed. Systems can either be under- or overfilled. Underfilled designs are the most common and do not waste available laser energy because the facet is sized such that the beam footprint on the facet never crosses over the edges of the facet during the full system scan angle. On the other hand, in an overfilled design the polygon facet is sized such that the beam completely fills the polygon facet over the entire full scan angle. Underfilled designs are preferred in many applications because there is less wasted energy and there is minimal diffraction from the facet edges. Overfilled designs have the one advantage that the system duty cycle can approach $100 \%$. The duty cycle is the ratio of the active scan time to the full facet time.

\subsection{POLYGON SIZE CALCULATION}

Once a system concept is chosen, and the optical design completed, the polygon size needs to be calculated. A few key parameters must be known in order to size the polygon:

1. Scan angle, $\theta$

2. Beam feed angle, $\alpha$

3. Wavelength, $\lambda$

4. Desired duty cycle, $C$

$\theta$ is the full extent of the active scan measured in degrees as illustrated in Figure 4.20. This value is usually in the range of $5-70^{\circ} . \alpha$ is the beam feed angle measured in degrees between the input beam to the polygon and the center of the scan exiting the polygon. It will be cost effective to keep this angle as small as possible in order to reduce polygon size. In certain scanner applications the beam feed angle is zero. The beam is brought in through a beamsplitter in the center of scan or at a slight angle relative to the exiting scanned beam. $\lambda$ is the operating wavelength expressed in microns and to be used in the calculation of the beam size on the polygon with a known desired spot size in the scan plane. $C$ is the duty cycle, which is the ratio of active scan time to total time. Duty cycles in the range of $30 \%-90 \%$ are common. However, the greater the duty cycle, the larger and more costly the polygon. With all conventional scan systems with the exception of monogon drum scanners some portion of the time will be spent transitioning from one facet to the next. We will assume that the design being considered is underfilled. This means that only one facet is being used to scan the image plane at any given time. 


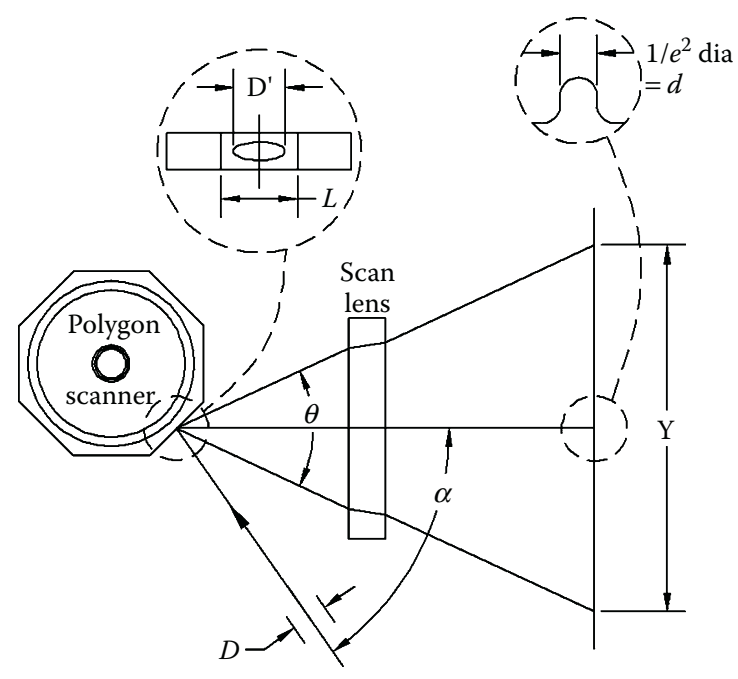

FIGURE 4.20

Illustration of scan angles.

The number of facets, $n$, to be used is a trade-off that needs to be addressed. The formula for the number of facets is given by:

$$
n=\frac{720 C}{q}
$$

If this equation produces a noninteger answer, this means that there is no exact solution to provide the duty cycle desired at the same time as the optical scan angle requirement is satisfied. A next logical step is to fix the number of facets to an integer value near the result from the previous calculation and fix either the scan angle or the duty cycle and solve for the remaining variable.

$$
C=\frac{n q}{720}
$$

For a writing application, once the duty cycle, scan angle, and number of facets are determined, the beam diameter $D$ incident to the facet can be calculated. The following formulas assume a Gaussian beam profile and the beam size defined at the $1 / e^{2}$ intensity points.

$$
D(\mathrm{~mm})=\frac{1.27 I F}{d}
$$

where $F$ is the focal length of the scan lens in $\mathrm{mm}$, and $d$ is the $1 / e^{2}$ beam diameter in the scan plane in microns.

The polygon can be sized without a scan lens by using the following formula:

$$
D(\mathrm{~mm})=\frac{1.27 I T}{d}
$$


where $T$ is the distance from the polygon to the focal surface in $\mathrm{mm}$, and $d$ is the $1 / e^{2}$ beam diameter on the focal surface in microns.

For a reading system, $D$ is a selected value based on the system-limiting aperture. The intensity profile across the diameter is no longer Gaussian but top hat instead.

Since the size of the facet depends on the actual beam footprint on the facet, the feed angle effect on $D$ must be taken into account. The value $D^{\prime}$ is the projected footprint on the polygon facet. It takes into account the truncation diameter and the cosine growth of the beam on the facet due to the beam feed angle. The formula for calculating the beam footprint is:

$$
D^{\prime}=\frac{1.5 D}{\cos (a / 2)}
$$

The calculations assume a TEM00 Gaussian beam that is truncated at the $1.5 \times 1 / e^{2}$ diameter. If the application can tolerate more clipping at the start and end of scan the polygon size can be reduced.

The length of the facet $(L)$ can be approximated from the beam footprint using the following: ${ }^{3}$

$$
L(\mathrm{~mm})=\frac{D^{\prime}}{1-C}
$$

The polygon diameter can now be approximated as follows:

$$
\text { Diam }_{\text {inscribed }} \oplus \frac{L}{\tan (180 / n)}
$$

If the polygon diameter is too large then there are three options. The first is to reduce the duty cycle and suffer a higher speed and burst data rate. The second is to reduce the beam feed angle. The third is to allow more intensity variation across the scan by reducing the 1.5 multiplier. This in turn, reduces the facet length.

\subsection{MINIMIZING IMAGE DEFECTS IN SCANNING SYSTEMS}

In order to design a scanning system that accurately reproduces information, knowledge of the types of artifacts that the scan system can produce and visibility thresholds of these artifacts is needed. The specifications required to reduce the artifacts to acceptable levels vary by application; for example, a prepress imager has different requirements from a laser printer. This section is written with writing applications in mind. Many of the defects associated with writing applications can also be present in reading applications.

\subsubsection{Banding}

Banding is one of the most common scan artifacts that will show up in scanning systems. Banding is a periodic variation in the line-to-line separation or intensity of the output. The human eye is very sensitive to periodic errors. The sensitivity is frequency dependent and great care must be taken to ensure that scan errors in the peak frequency range are minimized. ${ }^{4}$ In continuous tone and halftone printers the line-to-line placement errors need to be reduced to less than $0.5 \%$ of the line spacing. In other applications this can be as large as $10 \%-20 \%$ before banding becomes visible. 
The sources of banding include reflectivity variations, dynamic track errors between facets, mechanical vibrations, electrical noise and secondary axis translation errors. Polygon reflectivity variations can be easily eliminated by properly specifying the polygon coating such that these errors will not be visible. A specification of less than $1 \%$ variation of reflectivity on all facets will be adequate for all but the most demanding applications.

Either improving the polygon itself or compensating for the error can reduce dynamic track errors. Either approach will increase system costs so the trade-off between the brute force approach of improving the polygonal scanner must be weighed against the costs of additional system complexity.

Dynamic track error compensation can either be active or passive. Active techniques rely on using an active component to move the beam to compensate for the error whereas passive techniques rely on optics to minimize the errors. Active correction techniques will compensate for repeatable errors, but not errors that vary throughout the scan line. Passive techniques will compensate for both repeatable and nonrepeatable errors.

Active correction techniques are usually based on sampling the beam position errors perpendicular to the scan direction (cross scan) between scans and applying a beam steering correction in the system prior to the polygon to change the beam pointing. These techniques are used primarily in low-speed systems due to the frequency response limitations of the beam steering components. Active correction systems are rare because there is added mechanical complexity, higher cost, and the lack of correction for changes that occur during scan.

Passive correction techniques are quite common and the basic concept is illustrated in Figure 4.21. The polygon facet is reimaged with some magnification to the scan plane in the cross-scan axis. A cylindrical lens element is typically used to create a line focus on the polygon facet. The reimaging of this line in the cross-scan axis can be accomplished using a variety of components. Common methods include using a toroidal element near the polygon, a cylindrical lens near the scan plane, or a cylindrical mirror near the scan plane. ${ }^{5,6}$ These passive methods will provide a significant reduction, but not perfect compensation due to pupil shifting. The pupil shift is due to the fact that the polygon rotates about its center rather than rotating about the facet. The facet vertex changes during rotation so the object point moves in and out as the facet rotates.

Banding does not necessarily result from optical effects. Other sources such as vibration or electrical noise can contribute to banding. Mechanical vibrations can be introduced by the rotating device and amplified by the scan system platform. If the platform is not rigidly coupled to the image plane, then relative motion between the scanner and the image can result in a banding artifact.

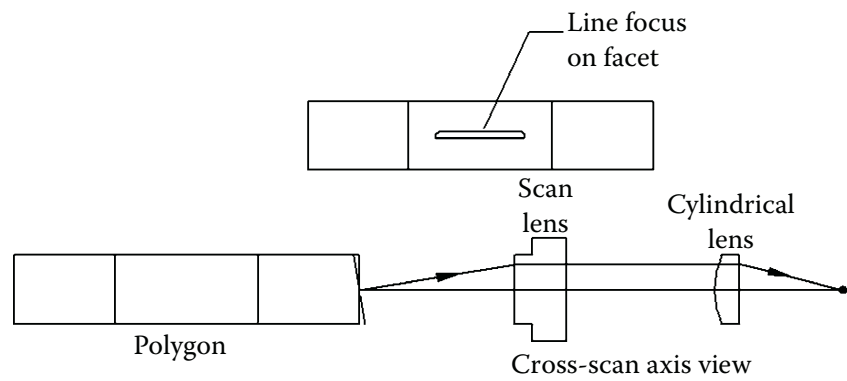

FIGURE 4.21

Passive cross-scan correction. 
Electrical noise can be generated by lasers or laser power supplies. It can modulate the laser output directly or it can affect the performance of an external modulation device such as an acousto-optic modulator. Continuous tone applications can be particularly sensitive to electrical noise. Repeatable noise on the order of $0.5 \%$ of peak power can be visible. The electrical noise can appear to be banding if the frequency is near to or a multiple of the revolution rate.

In most scan systems the second axis of the image is controlled by a mechanical device, such as a translation stage, direct drive rollers, or a belt drive. The velocity stability of this second axis must be specified to the same level of requirements as the scanner device. Velocity errors in this second axis directly impact the banding in the image.

This section has shown that there are a variety of sources of banding. Care must be taken in the design phase to properly specify all components that contribute to this problem since it can be difficult to isolate the root cause when this defect appears in a scan system.

\subsubsection{Jitter}

Jitter is the high-frequency variation in the pixel placement along the scan direction. Various systems can tolerate different levels of jitter before artifacts become visible. Output scanners that place a premium on pixel placement will typically require 0.1-pixel accuracy whereas visual image outputs can tolerate up to 1 pixel in many applications. Jitter has both random and repeatable components. Random jitter is visually less objectionable than periodic jitter.

Random jitter errors can be produced by the ball bearings used in most low-speed scanning systems. The magnitude of these errors is dependent on the inertia of the rotor, the ball bearings chosen, and the bearing mounting method. Errors are usually small enough not to be of concern. Aerostatic air bearings offer an alternative if the system is sensitive to the ball bearing errors.

Motor cogging with brushless DC motors can also create jitter errors that repeat once per revolution. The motor controller can reduce these errors with proper feedback rates (encoders or start of scan feedback), but they cannot be eliminated. One method to overcome these errors in low-speed applications is to retime the output data, based on actual scanner position information provided by an encoder. The only way to eliminate these errors is to find a motor with zero cogging torque. There is a class of toothless motors that have close to zero cogging torque. They are expensive, but may become more affordable as they further penetrate the market.

Polygon facet flatness variations result in a periodic jitter with a frequency of once per revolution or higher. The curvature causes small deviations in the angle of reflection from the facet. If the curvature of each facet varies, this causes the time between start of scan and end of scan to vary. A special case exists where there is no contribution to jitter if all facets have the same curvature. A facet flatness specification on the order of $\lambda / 8$ is adequate for most applications.

Facet radius variations in systems using post-objective scanning result in beam displacement in the scan plane. ${ }^{7}$ A facet radius variation specification of less than 25 microns is acceptable for most scanning applications.

\subsubsection{Scatter and Ghost Images}

There are many sources of scattered light and ghost images in an optical system. The majority of ghost images can be controlled through proper coatings and the placement of baffles. For example, if the strays are out of the plane of the scanned image an exit slit does 
a good job of eliminating them. Scan lenses can create problems with ghost images. The interior surfaces in these lens systems set up ghost images that are difficult if not impossible to eliminate with baffles. The antireflection coatings need to be high quality, reducing reflections to near zero.

As mentioned earlier in the chapter, the polygon surface can contribute to scatter. In extremely sensitive applications a diamond turned surface may produce too much scatter. This type of surface also produces a large percentage of near angle scatter that is difficult to baffle. A conventionally polished polygon produces wide-angle scatter and a much lower magnitude of total integrated scatter.

If the system contains an exit window or has a lens close to the scan plane, then the cleanliness of this element is important. Dust particles on this element can cause localized scatter in the scan plane since the spot is typically small at this point in the optical system. If repeated each scan, this will result in a line being produced down the image.

Adjacent polygon facets tend to be problematic in many systems where there is significant reflection from the target surface. The beam can find its way back through the system to the next facet. The problem with this type of stray light is that it will be on axis. The best solution is to tilt the scan plane a few degrees relative to the scan system so scan plane reflections are out of plane. Another solution is to mask the polygon sufficiently to leave only the active scan aperture open.

The time between scans when the beam is passing over the tips of the polygon is another source for scatter. Light will scatter from the tips of the polygon and from the side of scan lens mounts. Turning off the beam between scans and using a time interval counter to turn the beam on just prior to the start of scan sensor will eliminate this possible source of problems.

Acousto-optic modulators can produce several undesirable effects. Scatter from the crystal can limit the extinction ratio. Long decay times may result in tails when transitioning from black to gray in a continuous tone application. The crystals used can also suffer from sound field reflections that show up as ghost images. Working with an application engineer at the modulator supplier is the best way to avoid these issues from affecting a scan system.

\subsubsection{Intensity Variation}

Variations in laser intensity can produce a variety of image artifacts depending on the frequency of the variation. A slowly varying fluctuation is much less objectionable than a highfrequency variation. Whereas intensity variation on the order of a few percent may be tolerable over an entire image, local intensity variations may need to be controlled to less than $0.5 \%$.

Scan lens coatings and the coatings on any other elements located after the polygon can cause variations in the scan plane intensity across the scan. A transmission or reflection uniformity specification is needed to control this variable.

\subsubsection{Distortion}

Scanning systems typically employ a scan lens that has an F-theta characteristic. The lens distortion is controlled to produce image height that is proportional to the scan angle. This F-theta characteristic ensures linear scans with constant velocity. These lenses are not perfect but they do reduce the nonlinearity down to $0.01 \%-0.1 \%$ range. This is adequate for all but the most critical applications. These residual errors are repeatable, therefore; intensity compensation for dwell time differences or variable clocking schemes for pixel placement differences can be employed to remove the residual error. 


\subsubsection{Bow}

Bow is defined as the variation from straightness of a scan line. Bow is usually a slowly varying function across the scan. A considerable amount of bow can be tolerated before becoming visually objectionable. In most applications $0.05 \%$ of the scan line length of bow is an adequate specification. If the system is designed to have the beam brought in on the same axis as the scan then bow is caused by the errors in beam alignment. This can normally be adjusted to very fine levels and is therefore usually not a serious problem in a polygonal scan system. An equation for bow is given by:

$$
E=F \sin b\left[\frac{1}{\cos q}-1\right]
$$

where $F$ is the focal length of the scan lens, $E$ is the spot displacement as a function of field angle, $\theta$ is the field angle, and $\beta$ is the angle between the incoming beam and the plane that is perpendicular to the rotation axis. ${ }^{8}$

\subsection{SUMMARY}

This chapter has covered the components, performance characteristics, and design approaches for polygonal scanners and systems based on these scanners. This technology continues to evolve and thrives among increasing competition from other technologies both in writing and reading applications. I fully expect that the performance values that are stated in this chapter will be significantly improved on in the near future. However, the system level artifacts that a system designer must be careful to avoid tend to remain a constant. An in-depth knowledge of these artifacts and their root causes will help reduce development time for new systems.

\section{ACKNOWLEDGMENTS}

The author would like to thank Randy Sherman for his contributions to this chapter. Steve Lock and Jim Oschmann assisted with technical reviews of the chapter that were greatly appreciated. The author would also like to thank Luis Gomez of Lincoln Laser Company for providing the illustrations and Steven Stewart for the cover art. Photographs are courtesy of Lincoln Laser Company.

\section{REFERENCES}

1. Oberg, E. Machinery's Handbook, 23rd Ed; Industrial Press: New York, 1988; 196.

2. Bennett, J.M.; Mattsson, L. Introduction to Surface Roughness and Scattering; Optical Society of America: Washington, DC, 1989; 50-52.

3. Beiser, L. Design equations for a polygon laser scanner. In Beam Deflection and Scanning Technologies; Marshall, G.F., Beiser, L., Eds; Proc. SPIE 1454; 1991; 60-65. 
4. Bestenreiner, F.; Greis, U.; Helmberger, J.; Stadler, K. Visibility and corrections of periodic interference structures in line-by-line recorded images. J. Appl. Phot. Eng. 1976, 2, 86-92.

5. Fleischer, J. Light Scanning and Printing Systems. US Patent 3,750,189, July 1973.

6. Brueggemann, H. Scanner with reflective pyramid error compensation. US Patent $4,247,160$, January 1981.

7. Horikawa, H.; Sugisaki, I.; Tashiro, M. Relationship between fluctuation in mirror radius (within polygon) and the jitter. In Beam Deflection and Scanning Technologies; Marshall, G.F., Beiser, L., Eds; Proc. SPIE 1454; 1991; 46-59.

8. Hopkins, R.; Stephenson, D. Optical systems for laser scanners. In Optical Scanning; Marcel Dekker: New York, 1991; 46. 